Fehlheilungen

Paul Hochstein · Volkmar Heppert · Paul Alfred Grützner · Stefan Matschke • Andreas Wentzensen

Klinik für Unfall- und Wiederherstellungschirurgie an der Universität Heidelberg, BG-Unfallklinik Ludwigshafen

\title{
Rekonstruktion und Stabilisierung nach fehlverheilten Frakturen und Pseudarthrosen im Femurbereich mit Marknagel und anderen 0steo- syntheseverfahren (LISS-System)
}

e

\section{Zusammenfassung}

Die erfolgreiche Behandlung fehlverheilender Frakturen setzt eine konsequente Analyse der Heilungskomplikation voraus, um die geeignete weitere Therapie und das optimale operative Verfahren wählen zu können. Hypertrophe Pseudarthrosen des Femurschafts sind meist durch eine Verriegelungsmarknagelung nach Aufbohrung zu beseitigen. Torsionsdifferenzen werden bei funktionell bedeutsamer Größe bei früher Diagnose durch Neuplatzierung der distalen Verriegelungsbolzen beseitigt. Nach Frakturheilung sind eine offene Rotationsosteotomie oder die Korrektur mit einer Innensäge sinnvoll, stabilisiert wird mit Kondylenplatte oder Marknagel. Die Korrektur von Achsfehlstellungen erfolgt in Abhängigkeit von Fehlstellungslokalisation, Funktionsdefizit und Anforderungen an die Gelenkmechanik, häufig kann die Kondylenplatte erfolgreich eingesetzt werden. Für atrophe und septische Komplikationen sind zwar Behandlungsprinzipien aufgestellt worden, die individuelle Therapie erfordert jedoch ein umfangreiches erfahrungsorientiertes Wissen. Nicht selten versagen etablierte Vorgehensweisen. Bei Fehlheilungen aufgrund eingeschränkter Biologie des Knochens können neu entwickelte Osteosyntheseverfahren genutzt werden. Der Einsatz des LISS, u. U. kombiniert mit Spongiosaplastik, führt durch exzellente Stabilität und knochenferne Implantatlage zu Heilungen auch bei Worst-case-Verläufen.

\section{Schlüsselwörter}

Fraktur · Heilungsstörung · Fehlstellungen · Pseudarthrosen - Winkelstabile Implantate ehlheilungen nach Oberschenkelfrakturen sind in ihrer Häufigkeit deutlich von der Frakturlokalisation abhängig.

Schaftfrakturen haben eine niedrige Rate an verzögerten Frakturheilungen bzw. Pseudarthrosen. In der Literatur finden sich Angaben zwischen 1 und $5 \%$, eine eigene Analyse zeigte verzögerte Frakturheilungen nach Osteosynthese mit unaufgebohrtem Femurnagel (UFN) von $2 \%$. Auch korrekturrelevante Achsfehlstellungen sind eher die Ausnahme. Von Bedeutung sind allerdings bei Einsatz der gedeckten Marknagelung mögliche Torsionsdifferenzen. Eigene Überprüfungen (alle operierten Schaftfrakturen wurden innerhalb 1 Woche mit CT kontrolliert) zeigten Unterschiede zur gesunden Seite von $>20^{\circ}$ bei etwa 5\% aller Patienten. Bei der Aufarbeitung der zwischen 1978 und 1983 behandelten Patienten betrug die Häufigkeit 18\%, zu relevanten Auswirkungen kam es jedoch nur bei fehlendem Nulldurchgang.

Die Häufigkeit von Fehlheilungen und Fehlstellungen wächst bei metaphysennahen bzw. -beteiligenden Frakturen deutlich an. Komplikationsraten von $30 \%$ wurden v. a. bei distalen Oberschenkelfrakturen mitgeteilt.

Die Ursachen für Fehlheilungen und Fehlstellungen sind naturgemäß vielfältig, größere Untergruppen sind:

- ungeeignete Osteosyntheseverfahren

- verfahrensimmanente Komplikationen

- schwierige Frakturkonstellationen

- technische Implantationsschwierigkeiten
- biologische Probleme durch Weichteilund Gefäßschäden

Ein erfolgreiches Komplikationsmanagement beginnt mit der Versagensanalyse und führt unter Berücksichtigung mechanischer und biologischer Bedürfnisse zur weitergehenden Therapie. Wenn immer erreichbar, sollten alle Röntgenbilder, die den Verlauf dokumentieren, und alle Operationsberichte früherer Eingriffe zur Verfügung stehen.

Im Interesse des Verletzten liegt es, möglichst frühzeitig Komplikationen zu erkennen, damit diese zum optimierten Zeitpunkt beseitigt werden können. Dies bedeutet zwangsläufig, dass frühzeitige Korrekturen Teil des Therapieregimes sein sollten.

Es erscheint wenig sinnvoll, wenn trotz früh erkennbarer Fehlstellungen erst abgewartet wird, bevor dann doch eingegriffen und die Behandlung unnötig verlängert wird. Nur wenn durch eine frühe Korrektur der Heilungsprozess insgesamt gefährdet erscheint, ist ein Abwarten sinnvoll.

Indikationsstellung und Verfahrenswahl zur Therapie von Fehlheilungen wurden in der Vergangenheit immer

\footnotetext{
Dr.Paul Hochstein

Klinik für Unfall- und

Wiederherstellungschirurgie

an der Universität Heidelberg,

BG-Unfallklinik,

Ludwig-Guttmann-Straße 13,

67071 Ludwigshafen,

E-Mail:pshost@t-online.de,

Phone:0621-68102669, Fax:0621-68102400
} 
P. Hochstein - V. Heppert · P.A.Grützner -

S. Matschke $\cdot A$. Wentzensen

Medullary nailing and other internal fixation techniques (LISS system) for reconstruction and stabilization after malunion and nonunion of femoral fractures

\section{Abstract}

Malunion and nonunion after fracture present many difficulties in trauma surgery. Selection of the best therapy and operative techique requires careful analysis of the complications. Hyperthrophic nonunion of the femur shaft is mostly best treated with locking reamed nails. Significant differences in torsions after nailing can be corrected by repositioning of the distal interlocking screws if recognized sufficiently early. After fracture healing an open rotation osteotomy is necessary; stabilization can be achieved by means of medullary nails or a condylar plate. How a malalignment of the bone axis is corrected depends on the position of a malunion, the functional deficit caused, and the biomechanical demands on the joint. Condylar plates can often solve the problems in this region. There are also guidelines for the treatment of atrophic and infected nonunions (recommended procedures are aggressive debridement, autologous cancellous bone graft, and biological osteosynthesis), but the treatment of individual cases requires a exhaustive knowledge of and experience in this special field. It is quite common for even well-established procedures to fail. When the bone is biologically reduced and this is the reason for malunion newly developed techniques of internal fixation can be applied. The less invasive stabilization system (LISS), combined with autologous cancellous bone grafting if necessary, can result in healing even in a worst-case scenario.

\section{Keywords}

Fracture - Impaired healing - Malalignment . Nonunion · Angled implants
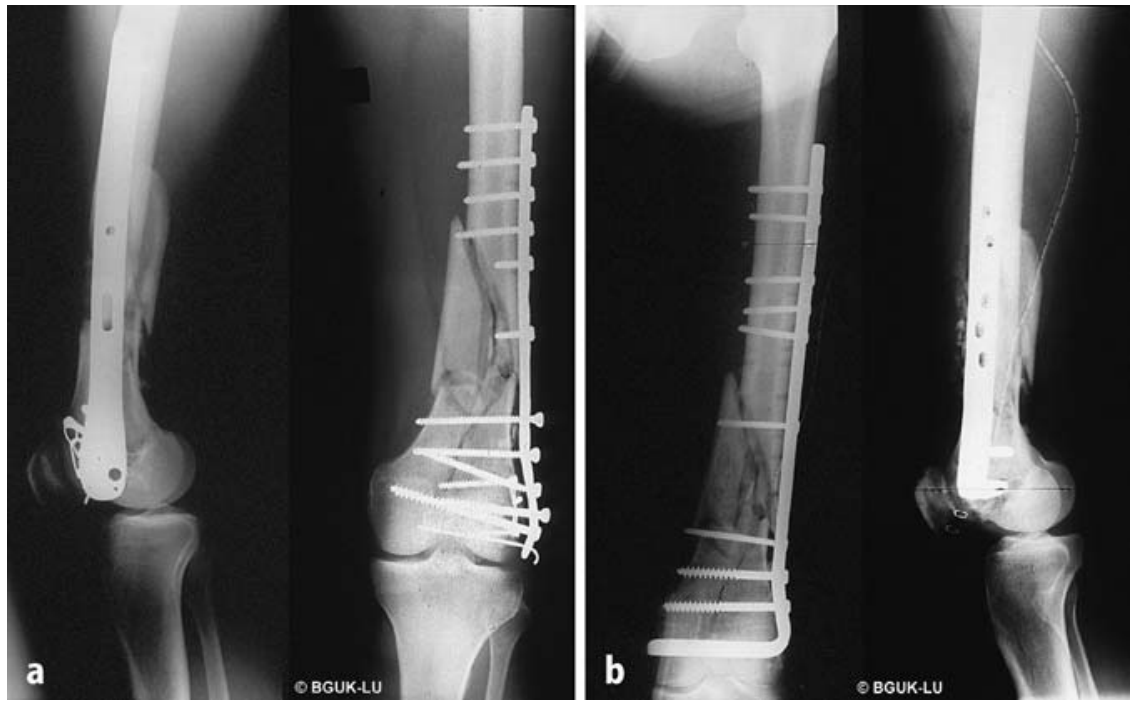

Abb. $1 \Delta$ a Instabile Osteosynthese bei Verwendung einer Abstützplatte, $b$ früher Verfahrenswechsel, Reosteosynthese mit Kondylenplatte

wieder ausführlich diskutiert. Bezüglich der Notwendigkeit einer raschen Behandlung manifester Pseudarthrosen bzw. infizierter Frakturen besteht kein Widerspruch. Eine Indikation zum Eingriff bei verzögerter Frakturheilung bedarf hinreichender Erfahrung in der Beurteilung einer bestehenden Heilungsprognose und der Einbeziehung individueller Gesamtumstände des Verletzten.

Dies gilt auch für die Korrektur von posttraumatischen Fehlstellungen. Über die Toleranzgrenzen besteht mittlerweile Einigkeit, jedoch sind Alter und Lebensumstände ebenso wie das spezielle Eingriffsrisiko in die individuelle Beratung des Verletzten einzubeziehen.

\section{Korrektur von Fehlstellungen in der Frühphase}

Gründe für sinnvolle frühe Korrekturen sind unzureichende primäre Behandlungsergebnisse, verursacht teils durch ungeeignete Osteosynthesetechniken bzw. Verfahrensauswahl (Abb. 1a), teils durch schwierige Fraktur- und Weichteilsituationen.

Weitere Indikationen entstehen bei sich sekundär entwickelnden Fehlstellungen bzw. drohenden Fehlheilungen (Abb. 2a). Dies setzt voraus, dass die radiologischen Verläufe unter Beachtung aller Röntgenbilder konsequent analysiert werden.

Die letzte größere Gruppe betrifft die dem Operationsverfahren immanenten Fehlstellungen. Gerade beim
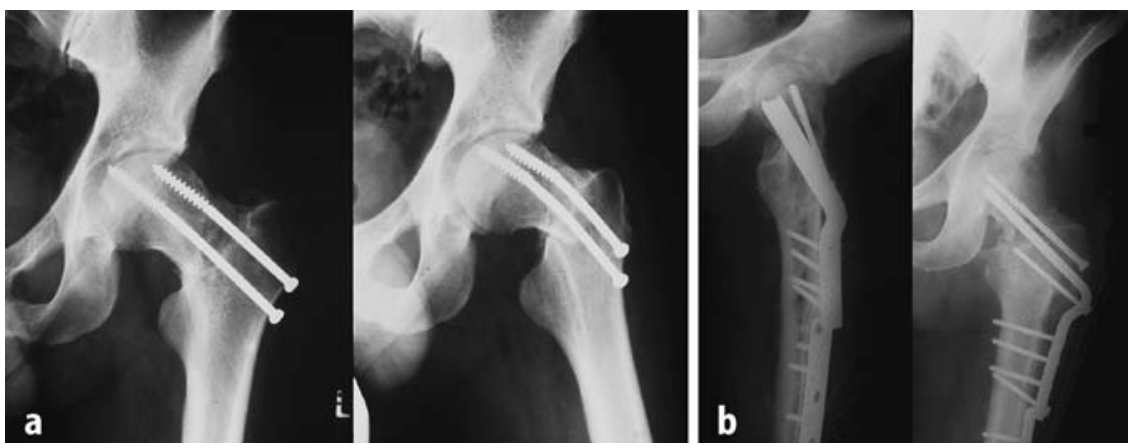

Abb. $2 \Delta$ a Zunehmende Fehlstellung nach Schenkelhalsfraktur, $b$ Verfahrenswechsel und valgisierende, einstellende 0steotomie zur Verbesserung der Frakturmechanik mit einer aufgebogenen Kondylenplatte 


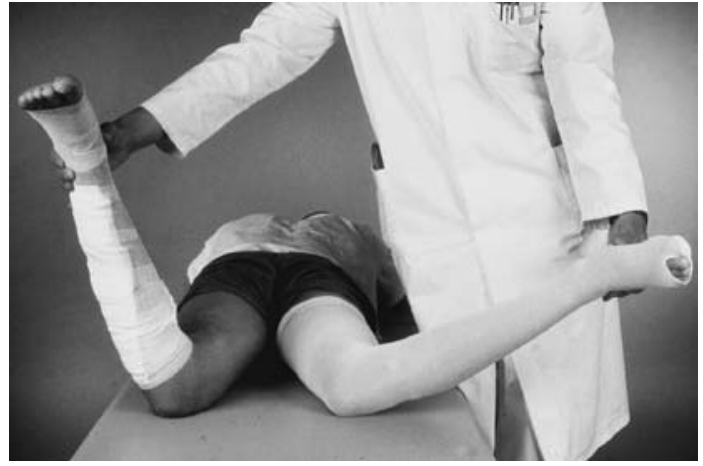

Abb. $3<$ Klinische Prüfung der Rotationsdifferenz

Einsatz gedeckter Osteosyntheseverfahren (Marknagel, eingeschobene Platten), die sich zur Stabilisierung von Oberschenkelfrakturen bevorzugt anbieten, ist mit operationsbedingten Rotationsdifferenzen (Abb. 3), die trotz aller gebotener Sorgfalt intraoperativ maskiert bleiben, $\mathrm{zu}$ rechnen. $\mathrm{Zu}$ fordern ist eine sorgfältige postoperative klinische Funktionsanalyse, um korrekturbedürftige Differenzen $\left(>20^{\circ}\right.$, fehlender Nulldurchgang bei der Hüftgelenkrotation) aufzudecken. Bei klinischen Unsicherheiten ist die rechnergesteuerte (z. B.CT, Sonographie) Bestimmung der Torsion erforderlich (Abb. 4).

Die Korrektur ungeeigneter Osteosynthesen richtet sich nach der individuellen Ausgangssituation, Ziel ist der Wechsel auf ein geeignetes Verfahren. Ähnliches gilt auch bei korrekturbedürftigen Fehlstellungen bzw. drohen- den Fehlheilungen: Im Individualfall gilt es zu entscheiden, ob ein Wechsel des Verfahrens (Implantat) sinnvoll ist oder ob das Implantat die notwendige Korrektur mit hinreichender Sicherheit zulässt (Abb. 1b, 2b).

Weniger problematisch ist die Verbesserung einer intraindividuellen postoperativen Torsionsdifferenz nach Marknagelung. Bei früher posttraumatischer Diagnostik kann das Problem durch eine Derotation und Neubesetzung der distalen Verriegelungsbolzen beseitigt werden. Bei späterer Diagnostik (etwa 2-3 Wochen nach der Versorgung) bleibt meist nur eine offene Korrektur, wobei abzuwägen ist, ob es nicht sinnvoller ist, einen Reeingriff erst nach Abschluss der Frakturheilung vorzunehmen, als den Heilungsprozess zu einem ungünstigen Zeitpunkt zu unterbrechen (Abb. 5).

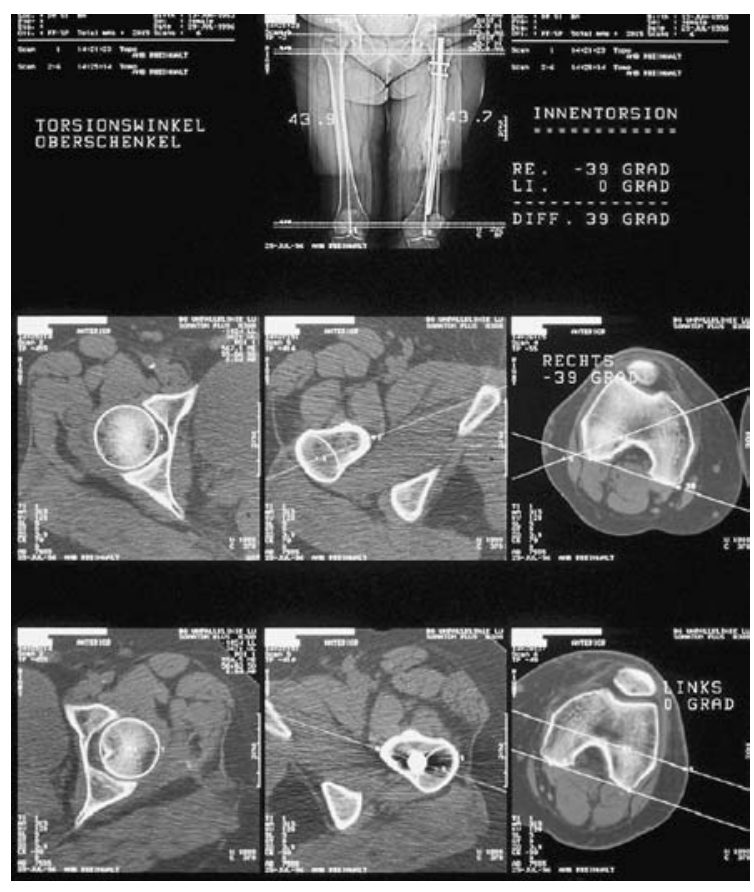

Abb. $4<$ Messung der Torsionsunterschiede mit CT

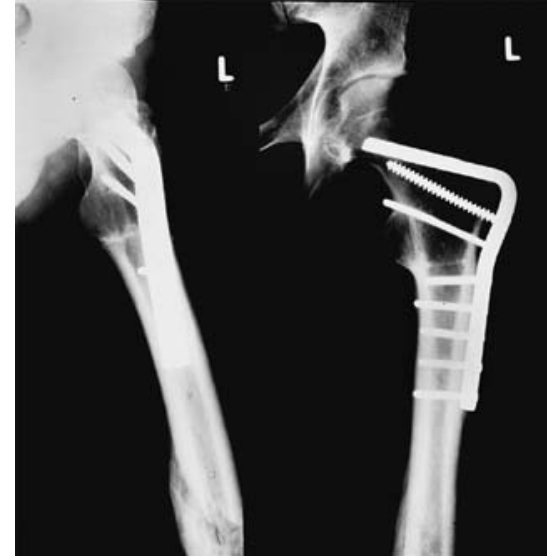

Abb. $5 \Delta$ Späte Korrektur einer Rotationsdifferenz unter Einsatz einer Kondylenplatte

\section{Korrektur von Fehlstellungen nach Abschluss der Heilung}

Die Indikation zur operativen Behandlung posttraumatischer Fehlstellungen richtet sich nach den individuellen Anforderungen des Verletzten (Alter, Aktivitätsgrad, etc.) und den Toleranzgrenzen für Achs- und Torsionsfehler (Tabelle 1).

Besteht eine Korrekturindikation, richtet sich das Verfahren (Ort der Korrektur) nach der Richtung und Lokalisation der Achsabweichung. Die meisten Fehlstellungen entstehen im metaphysären Bereich, hier ist die Osteotomie direkt im Bereich des Achsfehlers sinnvoll. Stabilisierungsprinzip in diesem Bereich sollte die Kompressionsosteosynthese sein, etablierte Verfahren wie die Kondylenplatte (alternativ DCS) bieten sich an. Marknagelverfahren sind eher weniger geeignet, da ein gedecktes Vorgehen (Hauptvorteil der Marknagelung) nicht möglich ist und die Verriegelung keine ausreichende Stabilität bietet.

$\begin{aligned} & \text { Tabelle } 1 \\
& \text { Toleranzen unfallbedingter Fehl- } \\
& \text { stellungen nach Frakturen der un- } \\
& \text { teren Extremität }\end{aligned}$
\begin{tabular}{ll} 
Fehlstellungen & Toleranz \\
\hline Varus & $>5^{\circ}$ \\
$\begin{array}{l}\text { Valgus } \\
\text { Torsion (Innendrehung- }\end{array}$ & $>10^{\circ}$ \\
$\begin{array}{l}\text { Außendrehung) } \\
\text { Kurvation } \\
\text { Verkürzung }\end{array}$ & $15^{\circ}-20^{\circ}$ \\
& $>15^{\circ}$ \\
& $2-3 \mathrm{~cm}$
\end{tabular}




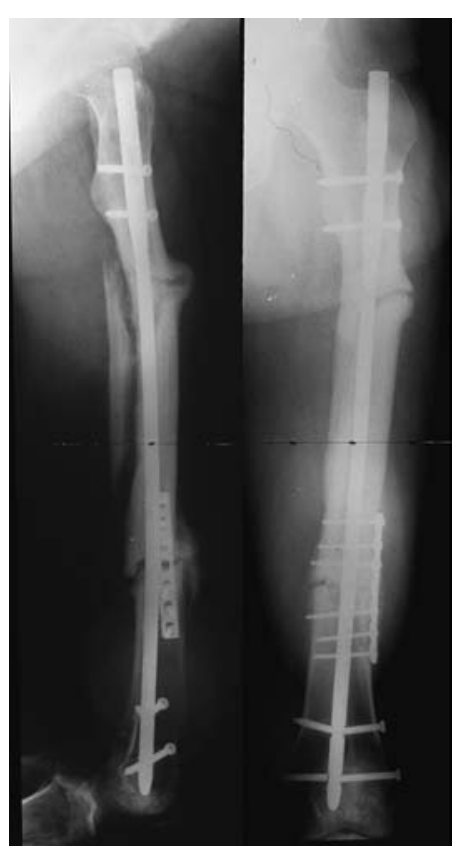

Abb. $6 \Delta$ Frakturheilungsstörung mit drohendem Einbruch des Nagels ins Kniegelenk, Ausheilung nach additiver Plattenosteosynthese, Kortikation und Spongiosaplastik

Lediglich bei einer Fehlstellung im Schaftbereich und liegendem Nagel ist die Korrektur mit Nagel sinnvoll. In diesen Fällen kann sich eine zusätzliche kurze Plattenosteosynthese über den Osteotomiebereich zur Sicherung der Korrektur und Kompression anbieten. Auch bei der Behandlung von Torsionsdifferenzen ist ein entsprechendes Vorgehen möglich. Nach Rückschlagen des Nagels erfolgt die Osteotomie, anschließend wird der Nagel nach Korrektur der Rotation wieder vorgeschlagen. Eine kurze gespannte Platte sichert die Rotation. Vorteil ist die sofortige Belastbarkeit.

Alternative Verfahren sind die Korrektur mit Innensäge und Marknagelosteosynthese oder die metaphysennahe Osteotomie und Stabilisierung mit Kondylenplatte (Abb. 5).

\section{Behandlung hypertropher Pseudarthrosen}

Die Behandlung hypertropher Pseudarthrosen ist häufig problemlos. Ziel der Therapie muss die Beseitigung der Restinstabilität sein, zusätzlich erfolgt ein Heilungsreiz durch Aufbohrung (bei Marknagelung) bzw. Dekortikation (bei Plattenosteosynthese).
Die Auswahl des Verfahrens wird durch die Lokalisation der Fehlheilung und ggf. durch das primär eingesetzte Osteosyntheseverfahren bestimmt. Hypertrophe Pseudarthrosen im Schaftbereich werden bevorzugt mit einem aufgebohrten Marknagel, metaphysennahe durch gespannte Kondylenplatten (evtl. DCS) bei gleichzeitiger Dekortikation erfolgreich behandelt. Spongiosaplastiken sind selten notwendig. In Einzelfällen kann auch bei frühzeitig erkennbarer hoher Instabilität und drohender Fehlheilung einer Marknagelosteosynthese eine zusätzliche Plattenosteosynthese (Abb. 6) zur Heilung führen.

Schwieriger als die prinzipielle Indikation zur Behandlung einer hyperthrophen Pseudarthrose erscheint die Entscheidung, wann ein erneuter Eingriff im Zeitraum einer "verzögerten“ Frakturheilung (zwischen 4 und 8 Monaten nach dem Trauma) bereits sinnvoll ist. Die Praxis belegt, dass bereits früh Fehlheilungen vorhersehbar sind, wenn sich unter vergleichender $\mathrm{Be}$ trachtung aller Röntgenverlaufsbilder kein Heilungsfortschritt mehr abzeichnet. Im Interesse des Verletzten liegt es, auch bereits vor der zeitlichen Manifestation einer Pseudarthrose ( $>7$ Monate nach Unfall) operativ einzugreifen, um die volle Funktion und Belastbarkeit so früh wie möglich zu erreichen. Ein zu langes Abwarten führt häufig nicht nur zu einer Verlängerung der Leidenszeit, sondern auch zu möglichen weiterge-
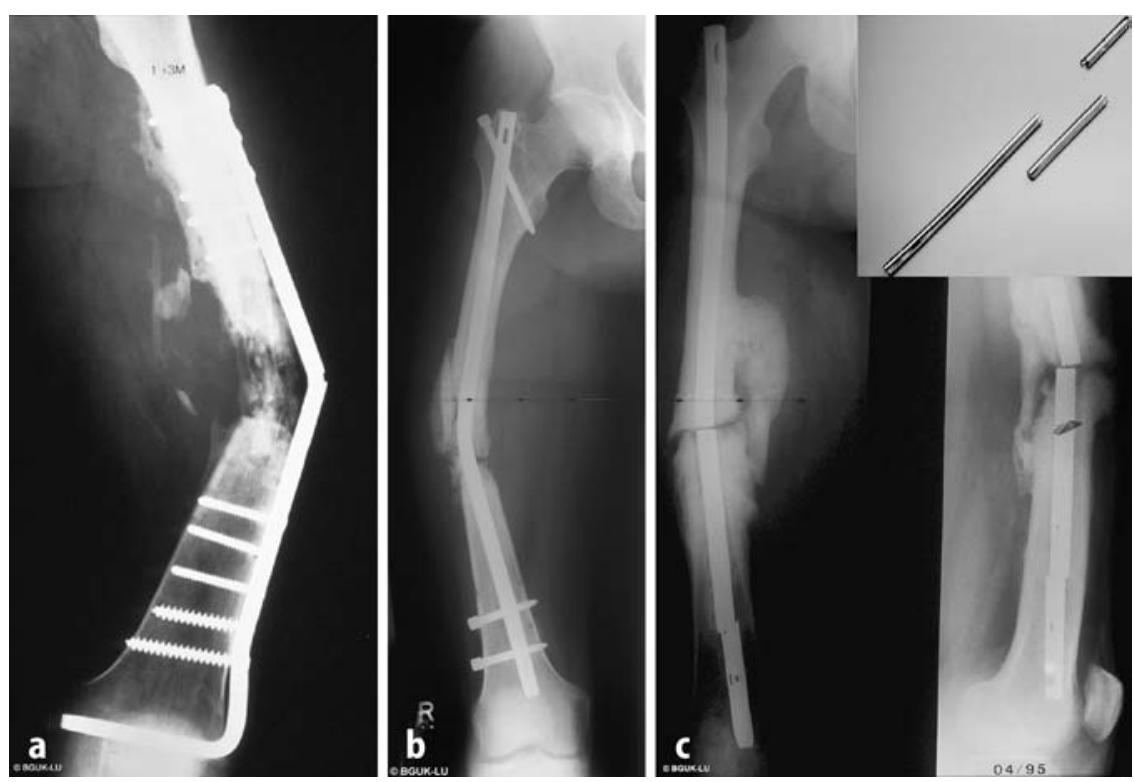

Abb. 7 A Implantatversagen bei Fehlheilungen henden Komplikationen wie Implantatbruch (Abb. 7, 8).

\section{Behandlung atropher Pseudarthrosen}

Schwieriger als die Behandlung hypertropher Pseudarthrosen ist die Therapie atropher Heilungsverläufe, da diese weniger durch mechanische Störungen, sondern aufgrund biologischer Defekte verursacht werden. Die Verbesserung biologischer Heilungsvoraussetzungen ist ungleich schwieriger als eine Verbesserung der Stabilität. Kommt es nicht zu einer knöchernen Heilung einer Fraktur einer belasteten Extremität, ist es nur eine Frage der Zeit, bis auch ideal eingesetzte Implantate versagen (Abb. 8).

Ziel eines Eingriffs bei atropher Pseudarthrose muss sein, Durchblutung und Heilungspotenz so zu unterstützen oder anzuregen, dass bei ausreichender Stabilität die knöcherne Heilung in Gang gesetzt wird.

Im Vordergrund der Behandlung steht meist die Implantation autologer Spongiosa. Bei der Auswahl des Osteosyntheseverfahrens darf nicht allein die mechanische Stabilität im Vordergrund stehen, berücksichtigt werden muss, dass durch das Implantat bzw. die Art der Implantation keine zusätzliche Verschlechterung der Durchblutung provoziert wird. Da in nahezu allen Fällen wegen der notwendigen Spongiosaanlagerung ein direkter Zugang zur Frak- 

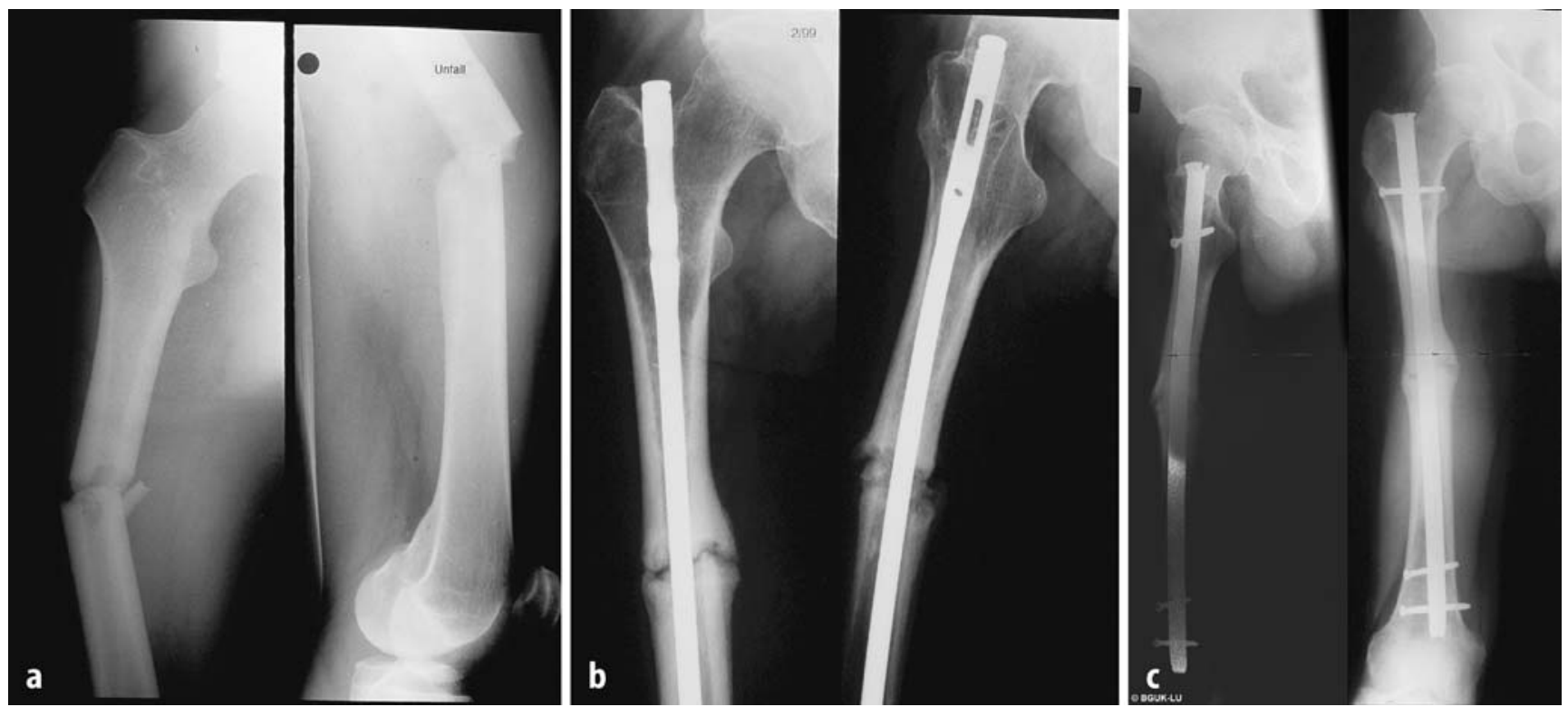

Abb. $8 \triangle$ a Fraktur am Unfalltag 10.9.1997, b 6.5.1998 Diagnosestellung einer Pseudarthrose, c Ausheilung Juni 1999, Aufbohrung und Nagelung erst im Februar 1999 nach Versuch einer Therapie mit Lithtrypser. Mehrkosten durch Verzögerung: 30.000 EUR

turzone erfolgen muss, bietet sich die Plattenosteosynthese als Stabilisierungsform an. In der ,klassischen “ Form als Kompressionsosteosynthese kommt es aber zu einer Reduktion der kortikalen plattennahen Durchblutung, dies führte in der Vergangenheit bereits zur Idee der Wellenplatte, bei der der Frakturbereich überbrückt wurde. Die neuartigen winkelstabilen Platten, die seit etwa 1997 zunehmende Verbreitung erfahren, ermöglichen heute durch verbesserte Verankerungsvoraussetzungen eine konsequente Weiterentwicklung dieses Konzepts. Aus der Erkenntnis hervorragender Heilverläufe beim Einsatz des LISS (less invasive stabilisation system) bei Problemfrakturen des Oberschenkels (Osteoporose und Prothesenrandfrakturen) wurde der interne Plattenfixateur auch bei atrophen Pseudarthrosen eingesetzt, bei denen konventionelle Implantate versagt hatten (Abb. 9). Es kam zu einer raschen Heilung auch im implantatnahen Bereich. Bei diesen Problemverläufen wurde allerdings das Prinzip der minimalinvasiven Implantation verlassen und nach Débridement und Spongiosaplastik der Plattenfixateur (LISS) mit Abstand zum Knochen „maximalinvasiv“ wie eine "normale“ Platte eingebracht (Abb. 9).

Alternativ zu den genannten Verfahren sind eine Resektion der atrophen
Pseudarthrose und eine entsprechende verkürzende Osteosynthese möglich. Nachteil ist naturgemäß der Längenverlust mit den resultierenden Einschränkungen für den Verletzten.

\section{Infizierte Pseudarthrose}

Die Therapie der infizierten Pseudarthrose ähnelt in späten Phasen der einer atrophen Pseudarthrose. Obligates primäres Ziel ist jedoch zunächst die Infektbeseitigung. Im Gegensatz zu einer frühen akuten Infektionstherapie ist bei einer infizierten Pseudarthrose immer von einer chronischen Osteomyelitis auszugehen. Eine Infekttherapie ist deshalb fast immer mit einer Resektion des betroffenen Knochens und mit einem entsprechenden Substanzverlust verbunden. Eine interne Osteosynthese in dieser Phase ist nicht sinnvoll, bis zur Infektberuhigung (reizlose Weichteile, Rückgang der laborchemischen Infektparameter) ist eine Stabilisierung mit Fixateur externe Verfahren der Wahl.

Erst in der 2. Phase kann ggf. wieder eine interne Osteosynthese eingesetzt werden, dies dann unter ähnlichen Kriterien wie bei einer primär aseptischen atrophen Pseudarthrose. Auch in diesen Fällen hat sich der Einsatz des LISS bewährt. Als Vorsichtsmaßnahme hat sich beim Verfahrenswechsel nach Beruhi- gung des Infekts neben der Implantation eines resorbierbaren Antibiotikaträgers das Konzept einer permanenten Dränage als günstig erwiesen. Wird im Abstrich, der am Knochen beim Wechsel auf eine interne Osteosynthese entnommen wird, ein Erreger nachgewiesen, wird die Dränage am Plattenlager als so genannte Dauerdränage belassen, bis das Implantat nach Konsolidierung frühzeitig entfernt werden kann (etwa 12-16 Wochen). Durch die permanente Sekretreduktion wird ein akuter Infekt verhindert und eine Heilung bei diesen Problemverläufen trotzdem ermöglicht.

\section{Resümee}

Nur eine frühzeitige Therapie von Fehlheilungen bzw. Fehlstellungen führt zu einer Verkürzung der Heilung mit der Folge einer erheblichen Leidens- und Kosteneinsparung.

Wenn im Sinn eines aktiven eigenen Komplikationsmanagements auch Bereitschaft für eine kritische Analyse eigener Behandlungsergebnisse besteht, kann häufig ein Zukunftsschaden verhindert bzw. zumindest gemindert werden.

Bereits frühzeitig müssen primäre bzw. sekundäre Fehlstellungen bzw. drohende Fehlheilungen erkannt und adäquat behandelt werden. Die Auswahl 

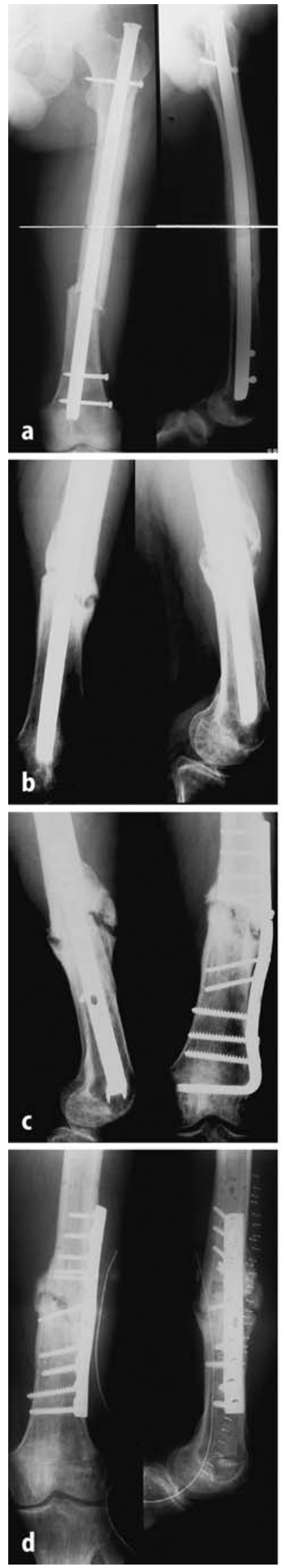

des Osteosyntheseverfahrens unterscheidet sich dabei nicht wesentlich von der Auswahl der Implantate bei der Primärversorgung. Sowohl intra- als auch extramedulläre Verfahren haben ihre am Einzelfall orientierte individuelle Bedeutung. Ein Verfahrenswechsel zwischen Marknagel- und Plattenosteosynthese sollte dabei in der Frühphase (etwa 6-9 Monate) möglichst vermieden werden, um keine weitergehende Reduktion der paraossären Durchblutung zu riskieren.

Im Komplikationsmanagement haben sich neben den Marknägeln vorwiegend winkelstabile Implantate bewährt. „Traditionelle"Verfahren wie Kondylenplatten bzw. DHS werden seit 1999 durch eine neue Implantatgeneration ergänzt. Diese Plattensysteme, die die Winkelstabilität durch die feste Verbindung zwischen Platte und Schraube erhalten, ermöglichen eine knochenferne Platzierung der Platte (als Fixateur interne) und tangieren die ossäre Durchblutung nicht unnötig, eine Idee, die zumindest im Ansatz bereits bei der Wellenplatte umgesetzt wurde.

In Zukunft wird es sich zeigen, ob durch die zunehmende Vielfalt lokalisationsspezifischer neuer Implantate (für jeden Knochenabschnitt ein anderes Implantat) ein Rückgang der Komplikationsrate erreicht und eine Komplikationstherapie erleichtert werden.

\section{Weiterführende Literatur}

1. Baumgart R, Kettler M,Zeiler C, Betz A, Schweiberer L (1997) Möglichkeiten der Knochendurchtrennung. Osteotomie- und Kortikotomietechniken. Unfallchirurg 100:797-804

2. Braun W, Markmiller M (1995) Müssen Achsfehler korrigiert werden:Die Grenzen physiologischer Belastung als Indikation zur Korrekturosteotomie, Hefte Unfallchirurg 257:653-663

3. Hierholzer G, Miller KM (1984) Korrekturosteotomien nach Traumen an der unteren Extremität. Springer, Berlin Heidelberg New York

4. Josten Ch (1995) Umstellungen "rund ums Knie". Hefte Unfallchirurg 257:675-680

5. Schatzker J (Hrsg) (1984) The intertrochanteric osteotomy. Springer, Berlin Heidelberg New York

6. Schweikert C-H, Rahmanzadeh R (1971) Femurschaft- und intertrochantere Osteotomie als Behandlungsprinzip des posttraumatischen Längenunterschieds. Hefte Unfallheilkd 107

7. Strecker W, Hoellen I, Keppler P, Suger G, KinzI L (1997) Torsionskorrekturen nach Marknagelosteosynthesen der unteren Extremität. Unfallchirurg 100:29-38

8. Strecker W (Hrsg) (1997) Posttraumatische Beindeformitäten. Analyse und Korrektur.Springer, Berlin Heidelberg New York

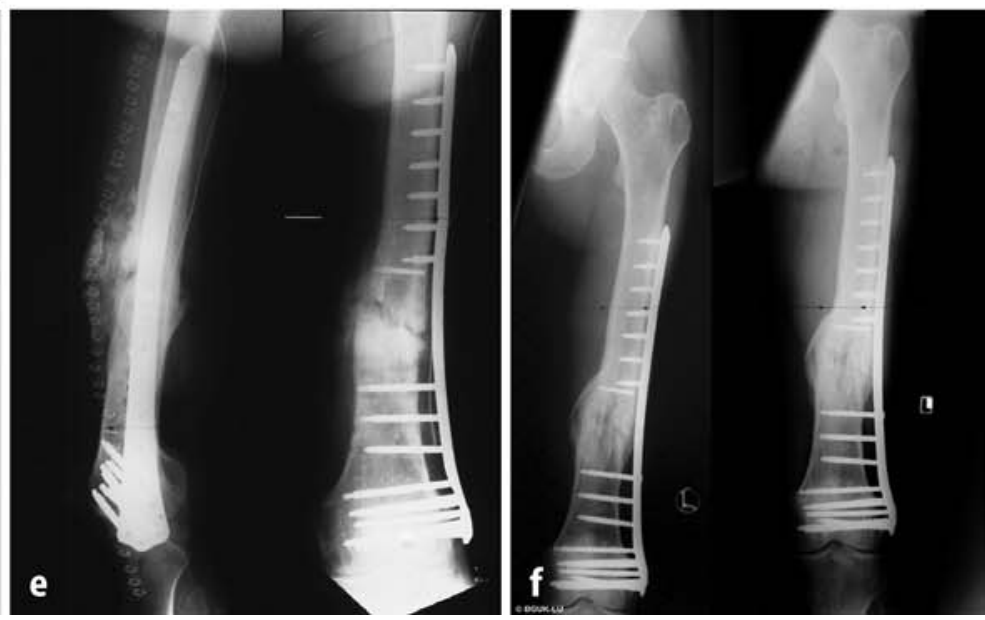

Abb. $9<$ a Fraktur nach Sturz vom Baum im 0ktober 1998, b Juni 1999, atrophe Pseudarthrose, c Juli 1999 gespannte Kondylenplatte, d November 1999: nach Implantatbruch Spongiosaplastik und Plattenosteosynthese, e,f Februar 2000 erneutes Implantatversagen, Implantation eines LISS, Ausheilung August 2000 